\title{
Crecimiento económico, precios y consumo de energía en México
}

\author{
Economic growth, price and power consumption in Mexico
}

\author{
Juan Marroquín Arreola* \\ Humberto Ríos Bolívar
}

Recibido: 27 enero 2016/ Aceptado: 24 agosto 2016

\section{Resumen}

El objetivo de este trabajo es evaluar empíricamente el papel que juegan los precios de la energía en el crecimiento económico de México. Para lo cual se expone un modelo de crecimiento endógeno de dos sectores que muestra la relación entre precio de energía con crecimiento del producto y consumo de energía. Los resultados muestran que la tasa de crecimiento de la producción y el consumo de energía se ven afectados negativamente por la tasa de crecimiento del precio de la energía.

Clasificación JEL: C33, O41, Q43.

Palabras Clave: Energía. Crecimiento endógeno. Panel.

\section{Abstract}

The aim of this paper is to empirically assess the role of energy prices on economic growth in Mexico. For this purpose, an endogenous growth model of two sectors showing the relationship between energy price growth in output and energy consumption is exposed. The results show that the growth rate of production and consumption of energy are negatively affected by the growth rate of energy prices.

\footnotetext{
* Escuela Superior de Economía del Instituto Politécnico Nacional. Plan de Agua Prieta \#66, Col. Plutarco Elías Calles, Del. Miguel Hidalgo, México D.F. C.P. 11340. Tel. 57296300 ext. 62037. Correo: juanmarro@gmail.com

** Escuela Superior de Economía del Instituto Politécnico Nacional. Plan de Agua Prieta \#66, Col. Plutarco Elías Calles, Del. Miguel Hidalgo, México D.F. C.P. 11340 . Tel. 57296300 ext. 62037. Correo: hriosb@hotmail.com
} 
JEL Classification: C33, O41, Q43.

Keywords: Energy. Endogenous growth. Panel.

\section{Introducción}

El papel de la energía en una economía plantea cuestiones importantes. Por ejemplo, los efectos de los precios de la energía tienen consecuencias para las economías en sus capacidades productivas internas, que a su vez tienen consecuencias para sus posibilidades de consumo y lo más importante, en su crecimiento y bienestar a largo plazo.

La importancia de la energía en el sistema económico comenzó a tomar relevancia con Georgescu-Roegen (1971), quien argumentó que la dimensión física de la producción económica requiere una atención más explícita en la teoría del crecimiento. Después de la primera crisis del petróleo en 1973-74, otros economistas comenzaron a formular funciones de producción dependientes de la energía que incluyen la energía y los materiales, además de la mano de obra convencional y los insumos de capital (por ejemplo, Tintner et al., 1974; Berndt y Wood, 1979).

El World Economic Outlook (2008) informó que mientras que el consumo de energía por unidad de PIB disminuyó en un $40 \%$ en los países avanzados, los países emergentes y en desarrollo, especialmente, son generalmente mucho más intensivos en energía. Esta evidencia sugiere que las perturbaciones del precio del petróleo tienen efectos económicos más graves en un país en desarrollo, que en una economía industrializada moderna.

Últimamente el sector energético ha recibido la atención, sobre todo de los responsables políticos. Gran parte de la atención se centra en la identificación de los canales por los que las fluctuaciones del petróleo influyen en el comportamiento de la economía, incluyendo su tasa de crecimiento, aunque sobre todo desde la perspectiva de los países avanzados (véase Barsky y Kilian (2004), para una revisión reciente).

La aparición de la crisis del petróleo en la década de 1970 estimuló el interés en el tema de la energía, generando una amplia investigación sobre cómo las fluctuaciones de precios de la energía como petróleo, gas, carbón y electricidad afectan a la economía. Es evidente que la importancia de estas consecuencias depende del grado de dependencia que se tenga sobre dichas fuentes de energía, su estructura de producción y su grado de integración financiera en la economía del país. 
La disminución de los precios del petróleo durante los últimos meses de 2014 y principios de 2015, y el temor de continuar así en los siguientes años, son causa de preocupación para los países productores de petróleo. Por lo tanto, dado el papel clave que desempeña la energía en las economías modernas, es importante entender los canales por medio de los cuales, las perturbaciones de precios del petróleo influyen en el rendimiento económico y el bienestar de las economías. Así pues, el objetivo de este trabajo es evaluar empíricamente el papel que juegan los precios de la energía en el crecimiento económico y en el consumo de energía en México. La hipótesis es que la baja del precio de la energía afecta de manera positiva al crecimiento económico.

En México, Petróleos Mexicanos (PEMEX) es el único productor de petróleo crudo, gas natural y productos refinados; por lo tanto, la producción de petróleo y gas natural depende de la empresa, y es de gran importancia tanto para el crecimiento económico de México, como para el abasto energético de EE.UU. La petrolera estatal sigue siendo una importante fuente de ingresos del gobierno y proporciona más de un tercio de los ingresos del gobierno federal.

Es evidente que el papel de la energía — en una economía - plantea preguntas importantes que merecen una mayor investigación. En particular, los efectos de las fluctuaciones de los precios de la energía tienen consecuencias importantes para sus capacidades productivas internas, sus posibilidades de consumo y, lo más importante, su bienestar. Los precios del gas, electricidad y carbón tienen un impacto directo en la actividad productiva de un país. Para abordar estas cuestiones, se expone un modelo de crecimiento endógeno basado en Berk y Yetkiner (2013), donde se relaciona la energía con el crecimiento y el consumo de energía. Este modelo realiza cointegración de panel para varios países, y el que se presenta, está centrado únicamente para el caso de México, estimando series de tiempo en lugar de panel. La principal contribución de este documento es la aplicación empírica. El documento se divide en seis secciones. En la segunda sección, se expone una revisión de literatura. En la tercera, se expone el modelo. En la cuarta sección, se muestran los datos y se establece la metodología utilizada. En la quinta sección, se realiza un análisis econométrico. Finalmente, se presentan las conclusiones.

\section{Revisión de la literatura}

En la literatura se pueden identificar varias investigaciones empíricas referentes al tema de precios de la energía y su relación con el crecimiento económico o con el consumo de energía, tanto en países desarrollados como en países en desarrollo. Toman y Jenelkova (2003) sostienen que la mayor parte de la literatura sobre la energía y el desarrollo económico, discute cómo el desarrollo 
afecta el uso de energía, y no al revés. Esta rama de la literatura considera el crecimiento económico como el principal motor de la demanda de energía, y que únicamente las economías avanzadas, con un alto grado de capacidad de innovación, pueden disminuir el consumo de energía sin reducir el crecimiento económico.

Stern y Cleveland (2004), por su parte, han hecho hincapié en el efecto de los cambios en la oferta de energía sobre el crecimiento económico, en los países desarrollados y en desarrollo. Si el suministro de energía se considera un insumo homogéneo para la función de producción, significa que las restricciones de política afectan el suministro de energía y, entonces, el desarrollo económico se verá perjudicado.

Augustini y Damilola (2015) estudian la relación entre consumo de energía, los precios del petróleo y el crecimiento económico en Nigeria; y, con base en sus resultados, recomiendan que se introduzcan políticas que promuevan el consumo de energía y el crecimiento económico. Una forma de lograr esto es a través de la adopción de un marco de fijación de precios de la energía conveniente, que toma conocimiento tanto de la presente como de las futuras generaciones.

Masih y Masih (1996) examinan los vínculos recíprocos entre el consumo de energía y el crecimiento económico, a partir de datos procedentes de Túnez, durante el período 1974-2011. El estudio sugiere que las políticas energéticas deben reconocer las diferencias que se presentan en el nexo entre el consumo de energía y el crecimiento económico, con el fin de mantener un crecimiento económico sostenible en Túnez.

Yu y Hwang (1984) analizan la causalidad entre el producto nacional bruto y el consumo de energía, utilizando datos de la economía de Estados Unidos para el período 1947-1979. También analizan la relación causal entre el consumo de energía y el empleo. Ellos no encuentran ninguna relación causal entre el producto nacional bruto y el consumo de energía, sin embargo, sí encuentran un ligero flujo unidireccional del empleo hacia el consumo de energía.

Ilhan, Alper y Huseyin (2010) utilizan los datos del panel de consumo de energía (CE), así como el crecimiento económico (PIB) de 51 países entre 1971 y 2005. Encuentran que existe la causalidad de Granger a largo plazo, que va desde el PIB al CE para los países de bajos ingresos, y no hay causalidad bidireccional entre el CE y el PIB de los países de ingresos medios. Los resultados de este estudio tienen importantes implicaciones políticas y se observa que esta cuestión todavía merecerá una mayor atención en futuras investigaciones. 
Kraft y Kraft (1978) prueban empíricamente la relación causal entre la energía y el producto nacional bruto; ellos encuentran que existe una relación constante e invariable entre el consumo de energía bruta y el producto nacional bruto, tanto de energía a producto como a la inversa.

Stern (1993) examina la relación causal entre el PIB y el consumo de energía para el período 1947-1990, en los EE.UU. Utiliza un modelo de vector autorregresivo multivariado, y no encuentra evidencia de causalidad entre el consumo de energía y el producto interno bruto.

Gómez y Rodríguez (2015) estudian la relación causal entre el consumo de electricidad y el crecimiento económico de México durante el periodo de 19712011. Aplican pruebas de raíz unitaria y causalidad. Los resultados muestran que el consumo de energía y el crecimiento económico son estacionarios y también, que hay una relación de causalidad que va desde el crecimiento económico hacia el consumo de electricidad. Por lo tanto, cualquier política de conservación de la energía tendría poco o ningún impacto en absoluto sobre el crecimiento económico en México.

Ciarreta y Zarraga (2010) investigan la causalidad lineal y no lineal entre el consumo de electricidad y el crecimiento económico en España para el período de 1971 a 2005. Utilizan la metodología de Toda y Yamamoto (1995) y Dolado y Lütkepohl (1996). También aplican prueba de causalidad de Granger en un vector autorregresivo en primeras diferencias. Encuentran causalidad lineal unidireccional que va del producto interno bruto real hacia el consumo de electricidad.

Bashier (2016) investiga las relaciones causales de corto plazo y largo plazo entre el consumo de electricidad y el crecimiento económico en Jordania entre 1976 y 2013, utilizando un modelo de rezagos distribuidos autorregresivos. Los resultados del modelo indican causalidad bidireccional entre las dos variables.

Dado los estudios mencionados anteriormente, en este documento se realizará, para el caso de México, un análisis de la relación existente entre los precios de la energía con el crecimiento económico y el consumo de energía.

\section{El modelo}

Para llevar a cabo el objetivo del trabajo, se expone un modelo de crecimiento endógeno de dos sectores, basado en Berk y Yetkiner (2013). El modelo parte de algunos supuestos, los cuales son los siguientes. 1) Se supone que hay dos sectores en la economía; el sector de bienes de consumo y el sector de bienes de 
inversión. 2) También se supone que hay dos tipos de factores de producción; el capital físico, $K$, y energía, $E$. La función de utilidad global del consumidor representativo en la economía se define, como:

$\left.U\left(C_{t}\right)=\int_{0}^{\infty} e^{-\rho t} u\left(C_{t}\right)\right) d t$,

donde la función de felicidad es

$u(C)=\frac{c^{1-\theta}-1}{1-\theta}$,

donde $C$ es el nivel de consumo, $\rho$ es la tasa subjetiva de descuento, $\theta$ es la elasticidad por consumir y $1 / \theta$ representa la elasticidad de sustitución intertemporal. La tecnología de producción de bienes de inversión se define, como:

$$
Z_{I}=B K_{I}
$$

donde $Z_{I}$, representa la producción en el sector de bienes de inversión, $B$ es la productividad total de los factores y $K_{I}$ es una variable de flujo, es decir, es una interpretación más amplia del capital físico usado en la producción de bienes de inversión.

El bien de consumo se produce a través de variables de flujo de capital físico $\left(K_{C}\right)$ y energía $(E)$, con rendimientos constantes a escala. La tecnología de producción se define de la siguiente manera:

$Z_{C}=K_{C}^{\alpha} E^{1-\alpha}$

Se supone que el total de stock de capital físico $K_{T}=\left(K_{I}+K_{C}\right)$, se emplea totalmente. El proceso de equilibrio en el sector de bienes de inversión de la ecuación de beneficios $\prod_{I}=p_{I} B K_{I}-N_{I} K_{I}$, resulta en:

$$
p_{I} B=N_{I}
$$

donde $N_{I}$, es la tasa de alquiler nominal (costo de uso) del capital físico en la producción de bienes de inversión y $p_{I}$ es el precio de los bienes de inversión. La maximización del beneficio del sector de bienes de consumo produce una función inversa de demanda para el capital físico (empleado en el sector) y la energía. En particular, la ecuación de beneficios nominal $\Pi_{C}=p_{C} K_{C}^{\alpha} E^{1-\alpha}-N_{C} K_{C}-P_{E} E$, resulta en:

$p_{C} \alpha K_{C}^{\alpha-1} E^{1-\alpha}=N_{C}$

$p_{C}(1-\alpha) K_{C}^{\alpha} E^{-\alpha}=P_{E}$ 
En las ecuaciones (4a) y (4b), $N_{C}$ es la tasa de alquiler nominal de capital físico en la producción de bienes de consumo, $P_{E}$ es el precio nominal de la energía y $p_{C}$ es el precio de los bienes de consumo. El precio real de la energía se define como $P_{R E}=\frac{P_{E}}{p_{C}}$,

y se considera que crece a una tasa constante, y que la oferta de energía es infinita en el precio de la energía dada.

La condición de no arbitraje implica que la tasa de alquiler del capital, en ambos sectores, debe ser igual. Por lo tanto,

$$
N_{I} \equiv N_{C} \Rightarrow p_{I} B=p_{C} \alpha K_{C}^{\alpha-1} E^{1-\alpha} \Rightarrow p B=\alpha K_{C}^{\alpha-1} E^{1-\alpha}
$$

En (5),

$$
p=\frac{p_{I}}{p_{C}}
$$

es el precio relativo de los bienes de inversión en términos de bienes de consumo. Entonces, el costo de uso real del capital (i.e. la tasa de alquiler) es $N N=p B=\alpha K_{C}^{\alpha-1} E^{1-\alpha}$. Una clara implicación de la ecuación (5) es que $\hat{p}=(\alpha-1) \hat{K}_{C}+(1-\alpha) \hat{E}$,

donde $\hat{p}, \hat{K}_{C}$ y $\hat{E}$ representan las tasas de crecimiento de los precios relativos de bienes de inversión $p$, capital $K_{C}$ y energía $E$ utilizado por el sector de bienes de consumo, respectivamente. La definición estándar del costo de uso del capital es la siguiente:

$$
N N \equiv(r+\delta-\hat{p}) p
$$

donde, $r$ es la tasa real de interés en términos del precio de bienes de consumo, $\delta$ es la tasa de depreciación del capital y $\hat{p}$ es la pérdida de capital, debido a cambios en los precios.

Para el equilibrio competitivo, se examina el problema de optimización del consumidor representativo. Para este fin, bajo los supuestos del modelo, el valor del Hamiltoniano es:

$$
H=e^{-\rho t} \frac{C^{1-\theta}}{1-\theta}+\lambda\left\{r S F+P_{R E} E-C\right\}
$$

En la ecuación (7), $S F$ representa el stock financiero del consumidor y $r$ es la tasa de interés real. Se asume que el consumidor recibe $P_{R E} E$, ya que ellos son 
tratados como el propietario de las reservas de recursos de energía. Las condiciones de optimización de primer orden, son:

$$
\begin{aligned}
& \frac{\partial H}{\partial C}=0 \Rightarrow e^{-\rho t} C^{-\theta}=\lambda \\
& \lambda=-\frac{\partial H}{\partial S_{F}} \Rightarrow \lambda=-\lambda\{r\} \\
& \dot{S}_{F}=\frac{\partial H}{\partial \lambda} \Rightarrow \dot{S}_{F}=r \dot{S}_{F}+P_{R E} E-C
\end{aligned}
$$

Además de estas condiciones, se debe satisfacer la condición de transversalidad, $\lim _{t \rightarrow \infty} \lambda(t) \dot{S}_{F}=0$. La solución de las ecuaciones (8a) y (8b) arroja que

$$
\frac{c}{c}=\frac{1}{\theta}\{r-\rho\}
$$

En el equilibrio, los activos financieros deben ser iguales al capital físico en una economía cerrada sin gobierno; $S_{F}=p(t) K(t)$. Usando esta información, se puede transformar la restricción presupuestaria del consumidor representativo. En primer lugar, $\dot{S}_{F}=\dot{p} K_{T}+p \dot{K}_{T}$. De $(4 \mathrm{~b})$, el precio real de energía es $P_{R E}=(1-\alpha) K_{C}^{\alpha} E^{-\alpha}$ y de (5) y (6) $r=B-\delta+\hat{p}$.

Por lo tanto,

$$
\dot{p} K_{T}+p \dot{K}_{T}=p K_{T}(B-\delta+\hat{p})+(1-\alpha) K_{C}^{\alpha} E^{1-\alpha}-K_{C}^{\alpha} E^{1-\alpha} p \dot{K}_{T}=p K_{T}(B-\delta)-\alpha K_{C}^{\alpha} E^{1-\alpha}
$$

Si se sustituye $p B$ por $\alpha K_{C}^{\alpha-1} E^{1-\alpha}$ debido a (5), y si se dividen ambos lados por $p$, se tiene que

$$
\dot{K}_{T}=(B-\delta) K_{T}-B K_{C}
$$

Por lo tanto, del problema de optimización del consumidor representativo se obtiene (9) y (10).

El modelo se resuelve a través de las condiciones de primer orden derivadas de los problemas de optimización de las empresas y consumidores representativos. En primer lugar, si se usa $r=B-\delta+\hat{p}$, obtenido a partir de las ecuaciones (6) y (9), se tiene

$$
\frac{\dot{c}}{c}=\frac{1}{\theta}\{B-\delta+\hat{p}-p\} .
$$

Luego, sustituyendo $\hat{p}=(\alpha-1) \hat{K}_{C}+(1-\alpha) \hat{E}$, de (5), se tiene 


$$
\frac{\dot{c}}{c}=\frac{1}{\theta}\left\{B-\delta+(\alpha-1) \hat{K}_{C}+(1-\alpha) \hat{E}-\rho\right\} .
$$

Como $\hat{C}=\hat{Z}_{C}=\alpha \hat{K}_{C}+(1-\alpha) \hat{E}$ debido a la ecuación (2),

$$
\begin{aligned}
& \alpha \hat{K}_{C}+(1-\alpha) \hat{E}=\frac{1}{\theta}\left\{B-\delta+(\alpha-1) \hat{K}_{C}+(1-\alpha) \hat{E}-\rho\right\} \Rightarrow \\
& (1-\alpha+\alpha \theta) \hat{K}_{C}+(1-\alpha)(\theta-1) \hat{E}=B-\delta-\rho
\end{aligned}
$$

Finalmente, como $\alpha \hat{K}_{C}-\alpha \hat{E}=\hat{P}_{R E}$, debido a (4b), se obtiene:

$$
\begin{aligned}
& \hat{E}=\frac{1}{\theta}\left(B-\delta-\rho-\frac{(1-\alpha+\alpha \theta)}{\alpha} \hat{P}_{R E}\right) \equiv g^{\prime} \\
& \hat{K}_{C}=\frac{1}{\theta}\left(B-\delta-\rho-\frac{(1-\alpha)(1-\theta)}{\alpha} \hat{P}_{R E}\right) \equiv g \\
& \hat{C}=\hat{Z}_{C}=\frac{1}{\theta}\left(B-\delta-\rho-\frac{(1-\alpha)}{\alpha} \hat{P}_{R E}\right) \equiv a g+(1-\alpha) g^{\prime}
\end{aligned}
$$

Donde $\hat{P}_{R E}$ es la tasa de crecimiento de los precios de la energía. Las ecuaciones $(11 a-11 c)$ implican que los precios de la energía tienen un impacto negativo sobre la tasa de crecimiento del consumo de la energía, como se muestra también en Zon y Yetkiner (2003). Nótese que $\frac{1-\alpha+\alpha \theta}{\alpha}>\frac{(1-\alpha)(1-\theta)}{\alpha}$ y que $\frac{1-\alpha+\alpha \theta}{\alpha}>\frac{1-\alpha}{\alpha}$. Como se supone que la condición: $B-\delta-\rho>\frac{(1-\alpha+\alpha \theta)}{\alpha}$ se mantiene, entonces, las tasas de crecimiento de las ecuaciones (11a-11c) son positivas. Se resuelve entonces el resto del modelo bajo este supuesto. En primer lugar, usando la igualdad $\hat{p}=(\alpha-1) \hat{K}_{C}+(1-\alpha) \hat{E}$, se puede mostrar que $\hat{p}=-\left(\frac{1-\alpha}{\alpha}\right) \hat{P}_{R E}$

Este resultado también puede ser expresado como ${ }^{1}: p(t)=p(0) e^{-\left(\frac{1-\alpha}{\alpha}\right) \hat{P}_{R E} t}$.

\footnotetext{
${ }^{1}$ Recordando que la tasa de crecimiento de los precios de la energía es constante. Se puede escribir el resultado también como: $p(t)=p^{\prime}(0)(q(t))^{-(1-\alpha)}, p^{\prime}(0)=p(0)(q(0))^{-(1-\alpha)}$
} 
Siempre que la tasa de los precios de la energía sea positiva, el precio relativo de los bienes de inversión en términos de los bienes de consumo, $p(t)$, se aproxima a cero. De la igualdad de $r=B-\delta+\hat{p}$, se obtiene:

$$
r=B-\delta-\left(\frac{1-\alpha}{a}\right) \hat{P}_{R E}
$$

Obviamente, la tasa real de interés y por lo tanto la tasa de crecimiento del nivel de consumo, $\hat{C}$, son positivos si y solo sí $B-\delta>\left(\frac{1-\alpha}{\alpha}\right) \hat{P}_{R E}$. Además, de la ecuación (8b) se tiene que

$$
\hat{\lambda}=-\left\{B-\delta-\left(\frac{1-\alpha}{\alpha}\right) \hat{P}_{R E}\right\}
$$

Como $r>0, \lambda$ debe aproximarse a cero. Luego, si se resuelve la ecuación (10) a través del método de factor de integración, resulta:

$$
K(t)=\frac{B K_{c}(0)}{B-\delta-g} e^{g t}+\text { cons } * e^{(B-\delta) t}
$$

Donde cons significa el término constante. Se puede fácilmente determinar el valor del término constante a través de la condición de transversalidad. En particular, sustituyendo los respectivos valores de $\lambda$ y $S_{F}=p K_{T}$ en la condición de transversalidad $\operatorname{Lim}_{t \rightarrow \infty}\left\{\lambda(t) S_{F}\right\}=0$, produce que el término cons debe ser cero. Además, para esto, la condición $B-\delta-g>0$ debe mantenerse para que la condición de transversalidad converja a cero en el límite ${ }^{2}$. En conclusión, la trayectoria del stock de capital total viene dado, por:

$$
K_{T}(t)=\frac{B K_{C}(0)}{B-\delta-g} e^{g t}
$$

Por lo tanto, el stock de capital total crece a la tasa $g$. Dado que el stock de capital social está definido exógenamente como $K_{T}(0) \equiv K_{0}=\frac{B K_{C}(0)}{B-\delta-g}$, para el modelo, se pueden determinar los valores iniciales de las variables de flujo, i.e. $K_{C}(0), K_{I}(0), E(0)$.

\footnotetext{
${ }^{2}$ Para $\theta>1, \frac{(B-\delta) \alpha(\theta-1)+\rho}{\alpha \theta+1-\alpha}$ es sin duda positivo. Si $\theta<1, \rho>(B-\delta) \alpha(\theta-1)$, debe mantenerse.
} 
Por último, se determina la trayectoria temporal del PIB real. Para ello, se tiene en cuenta que el PIB nominal $\left(\mathrm{PIB}_{\mathrm{N}}\right)$ y el PIB real en términos de bienes de consumo $(Z)$ se define, como:

$P I B_{N}=p_{I} Z_{I}+p_{C} Z_{C}$

$Z=p Z_{I}+Z_{C}$

Dado que $p=\frac{p_{I}}{p_{C}}$

Así pues, se puede demostrar que el PIB real es:

$Z=\operatorname{cons} 1 e^{[\alpha g+(1-\alpha) g] t}$

En (13), cons $1=p(0) Z_{I}(0)+\left(K_{C}(0)\right)^{\alpha}(E(0))^{1-\alpha}$, una colección de valores iniciales del modelo. En conclusión, el stock de capital físico total, inversión en capital y el consumo de capital, todos crecen a la tasa $g$. Por otro lado, la demanda de energía crece a la tasa $g^{\prime}$ y el PIB real y el consumo crecen a la tasa $\alpha g+(1-\alpha) g^{\prime}$, la cual es la tasa de crecimiento ponderada de energía y capital físico. Así pues, la tasa de crecimiento de los precios de la energía tiene un efecto negativo en las tasas de crecimiento.

\section{Datos y metodología}

Las variables utilizadas en el estudio, son: el crecimiento económico, el consumo de energía y precio de la energía ${ }^{3}$. Los datos del crecimiento económico son tomados de la sección de estadísticas del Banco de México en su página electrónica, los datos del consumo de energía y precio de la energía son tomados de las estadísticas del sistema de información energética de la Secretaría de Energía en su página electrónica. El crecimiento económico es producto del distinto peso y dinámica que tienen los sectores productivos en la economía, así como de la competitividad que registra la actividad económica. En la gráfica 1, se puede observar la evolución del crecimiento económico.

\footnotetext{
${ }^{3}$ Esta variable se construye como un promedio ponderado que incluye los precios del petróleo, electricidad gas y carbón.
} 


\section{Gráfica 1}

\section{Evolución del crecimiento económico}

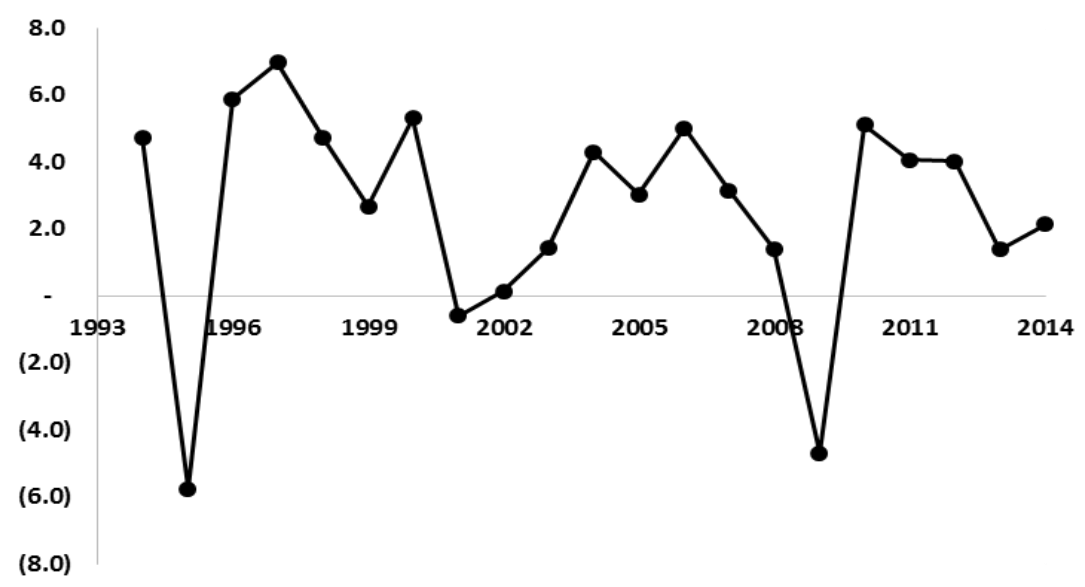

Fuente: elaboración propia con base en las estadísticas del PIB de Banco de México.

Cuando se habla de energía, la mayoría de la gente suele pensar solo en electricidad. Esta es solo una de las formas de energía que han sido explotadas durante muchos años. En combustibles convencionales, se incluyen: petróleo, gas natural, carbón y también se puede añadir la energía de los ríos, que se aprovecha para la energía hidroeléctrica.

Para la evaluación empírica se usa la técnica de cointegración de Johansen y Juselious (1990), quienes derivaron dos pruebas para cointegración: prueba de la traza (trace test) y máximo autovalor (Maximum Eigenvalue test). Las hipótesis que se plantean para las pruebas de la Traza se muestran en la tabla 1.

Las hipótesis que se plantean para las pruebas del máximo autovalor se pueden ver en la tabla 2: 
Gráfica 2

Evolución del consumo de energía

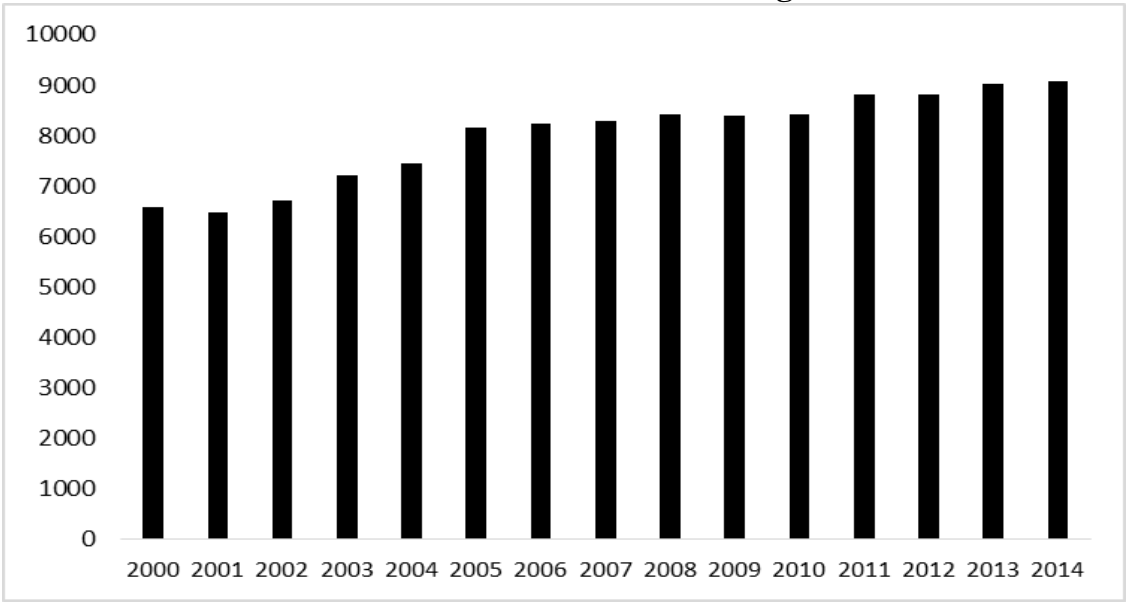

Fuente: elaboración propia con base en las estadísticas energéticas de la Secretaría de Energía.

Tabla 1

Prueba de la traza (trace test)

\begin{tabular}{|c|c|}
\hline Hipótesis & Regla de decisión \\
\hline $\begin{array}{l}\text { Ninguno } \\
\text { Ho: } r=0 \text { existen vectores de cointegración }\end{array}$ & $\begin{array}{l}\text { Se rechaza Ho cuando } \\
\text { el valor del estadístico } \\
\text { de la Traza o el Máximo } \\
\text { valor propio sea mayor } \\
\text { que el valor crítico } \\
\text { seleccionado. }\end{array}$ \\
\hline $\begin{array}{l}\text { Cuando más } 1 \\
\text { Ho: } \leq 1 \text { cuando mas existe un vector de cointegración }\end{array}$ & $\begin{array}{l}\text { Se acepta Ho cuando el } \\
\text { valor del estadístico la } \\
\text { Traza o el Máximo } \\
\text { Valor Propio sea menor } \\
\text { que el valor crítico } \\
\text { seleccionado. }\end{array}$ \\
\hline
\end{tabular}

Fuente: elaboración propia con base en Johansen y Juselious (1990). 
Tabla 2

Prueba del máximo autovalor (Maximum Eigenvalue test)

\begin{tabular}{ll}
\hline \multicolumn{1}{c}{ Hipótesis } & \multicolumn{1}{c}{ Regla de decisión } \\
\hline Ninguno & $\begin{array}{l}\text { Se rechaza Ho cuando el valor } \\
\text { del estadístico de la Traza o el }\end{array}$ \\
Ho: rango de cointegración $\mathrm{r}=0$ & Máximo Valor Propio sea mayor \\
$\mathrm{H}_{1}: \mathrm{r}=1$ rango de cointegración es igual a $\mathrm{r}+1$ & que el valor crítico seleccionado. \\
\hline Cuando más $\mathbf{1}$ & $\begin{array}{l}\text { Se acepta Ho cuando el valor del } \\
\text { estadístico de la Traza o el }\end{array}$ \\
$\mathrm{Ho}:$ el rango de cointegración es $\mathrm{r} \leq 1$ & Máximo Valor Propio sea menor \\
$\mathrm{H}_{1}:$ el rango de cointegración es $\mathrm{r}+2$ & que el valor crítico seleccionado. \\
\hline
\end{tabular}

Fuente: elaboración propia con base en Johansen y Juselious (1990).

\section{Análisis econométrico}

Para la realización del análisis econométrico, se hará uso de las ecuaciones fundamentales derivadas del modelo teórico, es decir, las ecuaciones 11a y 11c. Dichas ecuaciones se pueden reexpresar de la siguiente forma:

$$
\begin{aligned}
& \hat{E}=\beta_{0}+\beta_{1} \hat{P}_{R E} \\
& \hat{Z}_{C}=\gamma_{0}+\gamma_{1} \hat{P}_{R E}
\end{aligned}
$$

Donde $\beta_{0}=\gamma_{0}=\frac{1}{\theta}(B-\delta-\rho), \quad \beta_{1}=-\frac{1}{\theta} \frac{(1-\alpha+\alpha \theta)}{\alpha} \quad$ y $\gamma_{1}=-\frac{1}{\theta} \frac{(1-\alpha)}{\alpha}$.

Estas ecuaciones del modelo muestran una relación negativa entre el precio de la energía frente al crecimiento y consumo de energía; es decir, verificaremos econométricamente la hipótesis de que el aumento del precio de la energía afecta negativamente al crecimiento y consumo de energía.

En cualquier modelo que involucre series temporales, es necesario verificar el orden de integración de las variables implicadas. Para verificar si la serie es estacionaria, se pueden utilizar alternativamente diversas pruebas que, en la literatura, se conocen como prueba de raíces unitarias. En este estudio utilizaremos la prueba de Phillips-Perron (PP), ya que esta prueba extiende los contrastes de Dickey Fuller, con lo cual sus contrastes son más generales, y es posible considerar los contrastes de Dickey Fuller como un caso particular de Phillips-Perron. 
Las variables estimadas son: el consumo de energía $E$, el precio de la energía $P_{R E}$ y el crecimiento económico $Z_{C}$. Los resultados de la prueba de raíz unitaria de las variables de estudio se muestran en la tabla 3.

Tabla 3

Prueba Phillips-Perron (PP) de las variables

\begin{tabular}{ccccl}
\hline Variable & Prueba & $\begin{array}{c}\text { t } \\
\text { Phillips- } \\
\text { cerrón (PP) }\end{array}$ & $\begin{array}{c}\text { Valor crítico PP } \\
\text { al 5\% }\end{array}$ & $\begin{array}{c}\text { Estacionariedad/ } \\
\text { No } \\
\text { estacionariedad }\end{array}$ \\
\hline \multirow{2}{*}{$E$} & nivel & 0.74428 & -3.54874 & No estacionariedad \\
& 1a diferencia & -13.17423 & -3.431512 & Estacionariedad \\
& 2a diferencia & -31.63224 & -3.562625 & Estacionariedad \\
\hline$P_{R E}$ & nivel & -0.98234 & -2.46795 & No estacionariedad \\
& 1a diferencia & -10.22114 & -2.64162 & Estacionariedad \\
& 2a diferencia & -57.24473 & -2.98784 & Estacionariedad \\
\hline \multirow{2}{*}{$Z_{C}$} & nivel & -1.15101 & -4.43215 & No estacionariedad \\
& 1a diferencia & -18.44216 & -4.46244 & Estacionariedad \\
& 2a diferencia & -46.24242 & -4.96738 & Estacionariedad \\
\hline
\end{tabular}

Nota: Se utilizaron criterios de información de Akaike y Schwartaz para obtener el rezago óptimo, el cual fue 3. $E$ es el consumo de energía, $P_{R E}$ es el precio de la energía, $Z_{C}$ el crecimiento económico. Fuente: elaboración con base en resultados de la prueba PhillipsPerron.

Los resultados de la prueba PP muestran que los valores absolutos de los testadísticos calculados de todas las variables, son mayores que los valores críticos de primera y segunda diferencia, lo cual indica que se debe rechazar la hipótesis nula de que las series no son estacionarias y afirmar que las series sí son estacionarias. Una vez probada la estacionariedad de las variables se procede a realizar la prueba de cointegración. La variable consumo de energía es un promedio del consumo de energía, la variable precio de la energía también es un promedio que incluye los precios del petróleo, electricidad gas y carbón. La variable crecimiento económico es la tasa de crecimiento del producto.

Los resultados de la prueba de cointegración de Johansen-Joselius se muestran en la tabla 4. 
Tabla 4

Resultados de la prueba de cointegración de Johansen-Juselius

$\begin{array}{ccccc}\begin{array}{c}\text { Hipótesis del } \\ \text { número de } \\ \text { ecuaciones de }\end{array} & \begin{array}{c}\text { Estadístico } \\ \text { de la traza }\end{array} & \begin{array}{c}\text { Valor } \\ \text { crítico al }\end{array} & \begin{array}{c}\text { Estadístico } \\ \text { máximo }\end{array} & \begin{array}{c}\text { Valor } \\ \text { crítico al }\end{array} \\ \text { cointegración } & & \mathbf{5 \%} & \text { autovalor } & \mathbf{5 \%}\end{array}$

E vs $\mathbf{P}_{\text {RE }}$

$\begin{array}{ccccc}\text { Ninguna } & 17.45759 & 13.64647 & 29.68544 & 24.87664 \\ \text { Cuando más 1 } & 8.97546 & 5.987846 & 7.796565 & 4.321545\end{array}$

$\mathbf{Z}_{\mathbf{C}} \mathbf{v s} \mathbf{P}_{\mathbf{R E}}$

$\begin{array}{lllll}\text { Ninguna } & 12.88743 & 10.15485 & 11.79845 & 9.96657\end{array}$

$\begin{array}{lllll}\text { Cuando más } 1 & 7.47877 & 5.23214 & 5.97454 & 3.01213\end{array}$

Fuente: elaboración propia.

\section{Gráfica 3}

Función impulso-respuesta del precio de la energía sobre el consumo y el crecimiento del producto

Response to energy consumption to energy Price innovation

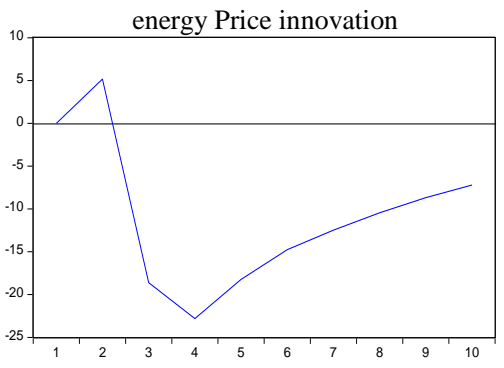

Response to economic growth to

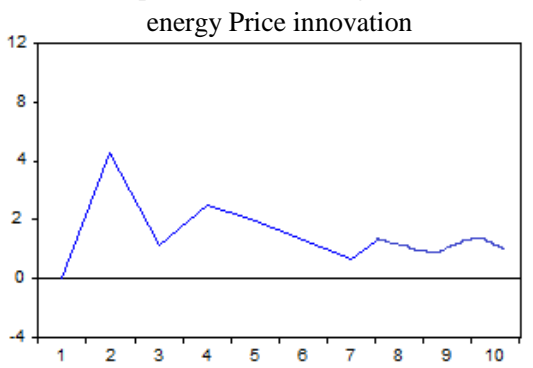

Fuente: elaboración propia con base al software Eviews 9.0.

La tabla 4 muestra que en las hipótesis de Ninguna y Cuando más 1, los estadísticos de la traza y máximo autovalor son mayores que los valores críticos. Esto indica que se rechaza la hipótesis nula de que no existe cointegración. Por lo tanto, los resultados confirman que sí existe la relación de largo plazo entre el consumo y los precios de la energía, y entre el crecimiento y los precios de la energía. 
Con objeto de conocer el impacto de los precios de la energía en el corto plazo, se realizó un análisis de impulso-respuesta. Como se observa en la gráfica 3, la función impulso-respuesta del consumo de energía, ante un shock en el precio de la energía, muestra un efecto negativo hasta el segundo periodo posterior, después de que sucede el shock y continúa siendo negativo en los periodos posteriores. Asimismo, la función impulso-respuesta del crecimiento económico ante un shock en el precio de la energía, también presenta un efecto negativo que se mantiene a lo largo del periodo.

\section{Conclusiones}

El objetivo de este trabajo fue evaluar empíricamente el papel que juegan los precios de la energía tanto en el crecimiento económico, como en el consumo de energía en México. Para ello, se presentó un modelo de crecimiento endógeno de dos sectores, donde se observa que los precios de la energía tienen un efecto negativo en el crecimiento económico. Asimismo, aplicando metodología de cointegración, se obtuvieron resultados que confirman el modelo teórico; es decir, los precios de la energía tienen efecto negativo sobre el crecimiento y sobre el consumo de energía. Los precios elevados de la energía perjudican principalmente a empresas que tienen un alto consumo de combustibles derivados del petróleo y gas; sin embargo, con la baja del precio del petróleo en los últimos meses, esas empresas podrían beneficiarse al obtener mayores ganancias por la reducción de costos, lo cual les permitiría crecer.

Así pues, dado los resultados del modelo, los altos precios de la energía provocan un menor crecimiento y consumo de energía; por lo tanto, habría de esperarse que una baja en el precio de la energía, contribuya a aumentar el crecimiento económico. Consecuentemente, las políticas económicas deben centrarse mucho más directamente en el aumento de la eficiencia energética y la innovación. Por lo tanto, el crecimiento económico futuro dependerá del progreso tecnológico, ya que de ello depende el uso eficiente de la energía. Sin embargo, el uso eficiente de la energía podría verse afectado por la demanda mundial de energéticos.

\section{Referencias}

[1] Augustine C. Osigwe y Damilola Felix Arawomo (2015). "Energy consumption, energy prices and economic growth: causal relationships based on error correction model". International journal of energy economics and policy, Econjournals, 5(2), 408-414.

[2] Barsky, R.B., and L. Kilian (2004). "Oil and the Macroeconomy Since the 1970s". Journal of Economic Perspectives, 18(4), 115-34. 
[3] Bashier, A. (2016). "Electricity consumption and economic growth in Jordan: bounds testing cointegration approach". European Scientific Journal, 12(1), 429-443.

[4] Berndt, E.R., Wood, D.O. (1979). "Engineering and Econometric Interpretations of Energy-Capital Complementarity". American Economic Review, 69, 342-354.

[5] Ciarreta, A. y Zarraga, A. (2010). "Electricity consumption and economic growth in Spain". Applied Economics Letters, 17(14), 1417-1421.

[6] Georgescu-Roegen, N. (1971). The Entropy Law and the Economic Process. Cambridge, Mass. Harvard University Press.

[7] Gómez, M. y Rodríguez, J.C. (2015). "Electricity consumption and economic growth: the case of Mexico". International Journal of Social, Behavioral, Education and Management Engineering, 9(8), 2803-2808.

[8] Istemi Berk and Hakan Yetkiner (2013). "Energy prices and economic growth: theory and evidence in the long run". Working papers in economics 13.

[9] Kraft J. and A. Kraft (1978). "On the relationship between energy and GNP”. Journal of Energy and Development, 3, 401-403.

[10] Ilhan, Ozturk, Alper, Aslan and Huseyin, Kalyoncu (2010). "Energy consumption and economic growth relationship: Evidence from panel data for low and middle income countries". Energy Policy, 38, 4422-4428.

[11] Masih A. M. M. and R. Masih (1996). "Energy consumption, real income and temporal causality: results from a multi-country study based on cointegration and error-correction modelling techniques". Energy Economics, 18, 165-183.

[12] Stern D. I. (1993). "Energy use and economic growth in the USA, A multivariate approach". Energy Economics, 15, 137-150.

[13] Stern, D.I., Cleveland, C.J. (2004). "Energy and Economic Growth". Rensselaer Working Papers in Economics No. 0410.

[14] Tintner, G., Deutsch, E., Rieder, R. (1974). A Production Function for Austria Emphasizing Energy. In Altman, FL, Kyn, $\mathrm{O}$ and Wagener, HJ (Eds), On the Measurement of Factor Productivities (151-169). Vandenhoek \& Ruprecht, Göttingen.

[15] Toman, M. and Jenelkova, Barbora (2003). Introduction of Energy Economics. Second edition. California USA, TTB Publication limited.

[16] Yu E. S. H. and B. Hwang (1984). "The relationship between energy and GNP: Further results". Energy Economics, 6, 186-190. 


\section{Apéndice}

Suponiendo que la energía es no renovable, entonces la condición de no arbitraje implica que el precio real de la energía debe aumentar a la tasa de interés real ${ }^{4}$ :

$$
\hat{P}_{R E}=r(t)
$$

La ecuación (1.1) se conoce como la regla de Hotelling en su forma más simple. Luego, se utiliza esta información en el modelo. Recordando que se obtuvo $\alpha \hat{F}_{C}-\alpha \hat{E}=\hat{P}_{R E}$ de la ecuación (4b), $r=B-\delta+\hat{p}$ de la ecuación (6) y $\hat{P}_{R E}=(\alpha-1) \hat{F}_{C}+(1-\alpha) \hat{E}$ de la ecuación (5). Por lo tanto,

$\alpha \hat{F}_{C}-\alpha \hat{E}=\hat{P}_{R E}=r=B-\delta+\hat{p}$

$$
\begin{aligned}
& \alpha \hat{F}_{C}-\alpha \hat{E}=B-\delta+(\alpha-1) \hat{F}_{C}+(1-\alpha) \hat{E} \\
& \hat{F}_{C}=B-\delta+\hat{E}
\end{aligned}
$$

Si esta información se usa en la ecuación (9), se obtiene,

$$
\begin{aligned}
& \frac{\dot{C}}{C}=\frac{1}{\theta}\{B-\delta+\hat{p}-\rho\} \\
& \frac{\dot{C}}{C}=\frac{1}{\theta}\left\{B-\delta+(1-\alpha)\left(\hat{E}-\hat{F}_{C}\right)-\rho\right\} \\
& \frac{\dot{C}}{C}=\frac{1}{\theta}\{\alpha(B-\delta)-\rho\}
\end{aligned}
$$

Por lo tanto,

$$
\begin{aligned}
& \hat{p}=-(1-\alpha)(B-\delta) \\
& r=\hat{P}_{R E}=\alpha(B-\delta) \\
& \hat{E}=\frac{1}{\theta}[\alpha(B-\delta)(1-\theta)-\rho] \equiv g^{\prime}
\end{aligned}
$$

\footnotetext{
${ }^{4}$ Supongamos que el mercado de energía es perfectamente competitivo y que la extracción no tiene costo. Bajo estos supuestos, la empresa representativa debería resolver el siguiente problema de maximización (Yetkiner y Van Zon, 2008; Gaitan et al., 2004):
}

$\operatorname{Max} \int_{0}^{\infty} P_{R E}(t) E(t) e^{-\int_{0}^{t} r(\tau) d \tau}$

sujeto a $\int_{0}^{\infty} E(t) d t \leq S_{0}$

$$
\operatorname{Lim}_{t-\infty}\left\{P_{R E}(t) e \int_{0}^{t} r(\tau) d \tau\right\}
$$

Donde $S_{0}$ es el stock inicial de energía no renovable. La solución del cálculo resulta en (12). 


$$
\hat{F}_{C}=\frac{1}{\theta}[(B-\delta)(\theta+\alpha(1-\theta))-\rho] \equiv g
$$

Dado que $B-\delta>0$ para una tasa de interés real positiva y tasa de crecimiento de los precios de la energía, $\theta$ debe ser menor que 1 . De lo contrario, la demanda de energía podría estar disminuyendo en el tiempo. Como $\theta+\alpha(1-\theta)>0$ es siempre verdadero, la tasa de crecimiento del stock físico empleado en el sector de bienes de consumo es positiva siempre y cuando, $(B-\delta)(\theta+\alpha(1-\theta))>\rho$. 